\title{
Smart hexagonal truss systems exhibiting negative compressibility through constrained angle stretching
}

\author{
Joseph N Grima ${ }^{1,2,5}$, Roberto Caruana-Gauci ${ }^{1}$, \\ Krzysztof W Wojciechowski ${ }^{3}$ and Kenneth E Evans ${ }^{4}$ \\ ${ }^{1}$ Metamaterials Unit, Faculty of Science, University of Malta, Msida MSD 2080, Malta \\ 2 Department of Chemistry, Faculty of Science, University of Malta, Msida MSD 2080, Malta \\ ${ }^{3}$ Institute of Molecular Physics, Polish Academy of Sciences, M Smoluchowskiego 17, 60-179, Poznań, \\ Poland \\ ${ }^{4}$ College of Engineering, Mathematics and Physical Sciences, University of Exeter, Exeter EX4 4QF, \\ UK \\ E-mail: joseph.grima@um.edu.mt and auxetic@um.edu.mt
}

Received 20 May 2013, in final form 10 June 2013

Published 22 July 2013

Online at stacks.iop.org/SMS/22/084015

\begin{abstract}
Negative compressibility is the ability to expand in at least one dimension rather than shrinking upon the application of an externally applied hydrostatic pressure. It is shown that, contrary to current perception, negative linear compressibility may be obtained from re-entrant hexagonal truss systems of specific geometric features which deform through non-equal changes in the lengths of the cell walls when deforming through a constrained angle stretching rather than other modes of deformation (such as flexure or hinging, modes of deformation that also lead to auxetic behaviour in honeycombs). Negative compressibility is predicted in the vertical direction for particular re-entrant geometries of this smart hexagonal truss system when the vertical ribs are much stiffer than the inclined ribs.
\end{abstract}

(Some figures may appear in colour only in the online journal)

\section{Introduction}

Cellular honeycombs with hexagonal repeat units in both their conventional and their re-entrant forms have been thoroughly studied and analysed in recent years, not only because such systems can afford some very interesting macroscopic properties, but also because such honeycomb systems can have a variety of applications. Some anomalous, yet highly desirable, macroscopic properties exhibited by such honeycombs include negative Poisson's ratio (auxetic behaviour, i.e. the property of becoming wider rather than thinner when uniaxially stretched [1-9]) and the property of negative linear compressibility (i.e. the ability to expand in at least one dimension rather than shrinking upon the application of an externally applied hydrostatic pressure [9-21]). Uses

5 www.um.edu.mt/science/metamaterials. of such honeycombs are equally diverse and range from their use in the manufacture of sandwich plates [22] to reinforcement in network embedded composites [2]. In recent years, analytical models have been developed to predict the mechanical behaviour of such cellular systems when they deform through various mechanisms, which include flexure, stretching and/or hinging of the cell walls [3-7]. These models can elucidate the necessary conditions for a system to exhibit the desired property. For example, in Gibson and Ashby's pioneering study, it was shown that for re-entrant honeycombs deforming through flexure of the cell wall (flexure mechanism), the honeycomb expands in the lateral direction when uniaxially stretched, i.e. a negative Poisson's ratio is observed [3, 4]. On the other hand, for non re-entrant honeycombs, a negative Poisson's ratio is observed if they deform through stretching of the ribs $[5,6]$. More recently, it was shown that honeycombs and other 
cellular solids deforming through changes in angles between the cell walls (hinging mechanism) may exhibit the rare property of negative linear compressibility (NLC) [16, 18]. In the latter case, negative linear compressibility is observed when certain conditions are satisfied through a mechanism that is not dissimilar to other main mechanisms known so far to be responsible for NLC, such as that employed by Baughman et al to explain the predicted NLC in some carbon allotropes [10], by Fortes et al to explain the experimentally measured NLC in methanol monohydrate [15] and by Cairns et al to explain the giant NLC response measured in the $\alpha$-polymorph of zinc dicyanoaurate [19]. These conditions include the requirement that the system is non-porous, such that the fluid that is exerting hydrostatic pressure does not permeate through the ribs of the structure (i.e. the hydrostatic pressure is exerted on the external sides of the honeycomb), and that the hexagonal system predominantly deforms, as wine-rack type structures typically do, through changes in the angles between the ribs of the structure (idealized hinging mechanism) rather than, for example, through stretching of the cell walls in the manner described by Masters and Evans [6]. In fact, it is currently perceived that mechanisms that involve fixed-direction stretching of cell walls rather than changes in the angles subtended between the cell walls nullify any mechanism-induced NLC properties, something that may explain why NLC is such a rare property in real materials.

Here, we show that there are no convincing arguments that prohibit NLC from mechanisms that only involve constrained angle stretching of cell walls rather than angle changes and we show that such behaviour may indeed be obtained from hexagonal truss systems having particular geometries if the different ribs in the structure are pre-designed to stretch to different extents (i.e. the ribs have different stretching stiffness constants).

\section{Model}

The system modelled here is based on figure 1 and may be described as an infinitely tessellated hexagonal truss system having the unit cell as shown in figure 1(a), where $l$ is the length of the inclined ribs, $h$ is the length of the vertical ribs and $\theta$ is the angle that the inclined ribs make with the horizontal direction. This angle $\theta$ assumes negative values in the range $-90^{\circ}<\theta<0^{\circ}$ for re-entrant hexagonal truss systems and positive values in the range $0^{\circ}<\theta<90^{\circ}$ for non re-entrant hexagonal truss systems, as defined in figure 1(a), with the unit cell parameters of the system in the $O x_{1}$ and $O x_{2}$ directions respectively being given by

$$
\begin{gathered}
X_{1}=2 l \cos (\theta) \\
X_{2}=2(h+l \sin (\theta)) .
\end{gathered}
$$

Whilst for $0^{\circ}<\theta<90^{\circ}$, the parameters $h$ and $l$ can assume values $h \geqslant 0$ and $l>0$, for $-90^{\circ}<\theta<0^{\circ}$, one also requires that

$$
h+2 l \sin (\theta)>0
$$

so as to ensure that the tips of the system do not overlap and hence the system is physically realizable as a $2 \mathrm{D}$ planar
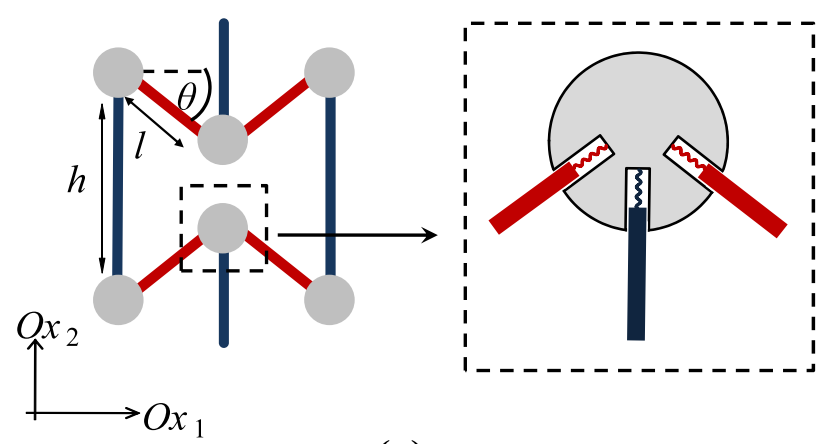

(a)

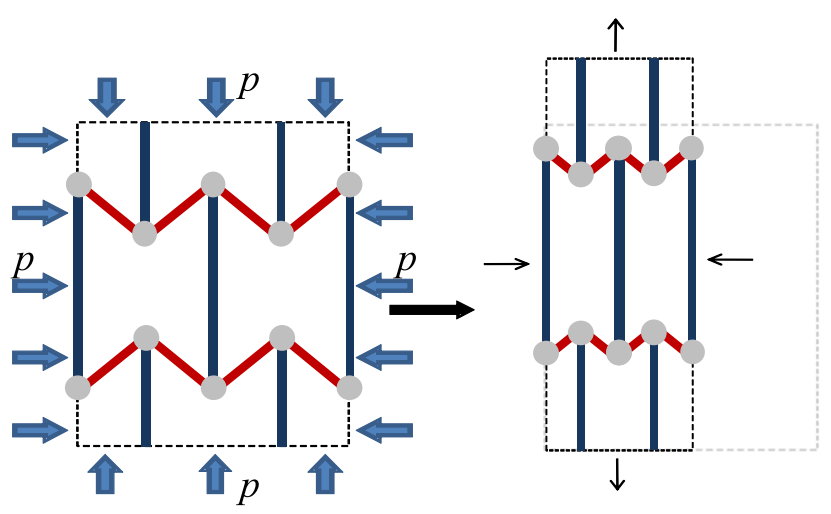

(b)

Figure 1. (a) The unit cell of the honeycomb geometry in its re-entrant configuration. Differently coloured ribs have different stretching stiffness constants. The detail shows how the ribs of this system are considered to be made up (as in [23]). (b) Application of a hydrostatic pressure exerted on the outside of such a system deforming through a constrained angle stretching mechanism results in negative linear compressibility $\beta_{2}$ along the $O x_{2}$ direction.

structure. Note that the system where $0^{\circ}<\theta<90^{\circ}$ and $h=0$ corresponds to a system with rhombic shaped cells rather than hexagonal and is termed as a wine-rack system.

It is assumed that the hexagonal truss system deforms in an idealized manner through constrained angle stretching of the ribs with the complete exclusion of any other deformation mode such as flexure or changes in the angles between the ribs. In other words, the only permissible way in which the system may deform is through change in length of the cell ribs, hence the term 'constrained angle stretching'. Also, it will be assumed that the hydrostatic pressure is exerted on the outside of the system, hence if one is exerting a pressure through means of a fluid, for the presented mechanism to work, the system has to be non-porous to the fluid particles (as discussed in [18]). More importantly, it shall also be assumed that the vertical ribs in the structure are pre-designed to have a stiffness constant $k_{\mathrm{s}}^{h}$ which is independent of and may be different from the stiffness constant $k_{\mathrm{s}}^{l}$ of the inclined ribs, such that the vertical and inclined ribs may stretch to different extents. Such a model may be considered as a generalized version of the idealized stretching mechanism as proposed by Masters and Evans [6] and is based on the mechanism employed by Wojciechowski and co-workers in their work on hexagonal systems [23, 12], which can also be used as 
discussed here. The concepts presented in this work may be implemented by, for example, having a system constructed from rigid ribs which are attached to a three-slot node by means of springs such that the ribs can slide in and out of the aforementioned slots, but cannot change the angle between them, as in figure 1. Clearly this system could become unstable when the elongation corresponds to the length of the slots in which the ribs are placed, i.e. the ribs slide out of their respective slots. Such unstable scenarios may be easily avoided by the practical expedient of preventing the ribs from extending this far.

For the systems discussed here, i.e. ones where deformations are only through a constrained angle stretching mechanism, the on-axis strains in the $O x_{i}$ direction for loading by an infinitesimally small stress $\mathrm{d} \sigma_{j}$ in the $O x_{j}$ direction may be defined as

$$
\mathrm{d} \varepsilon_{i}^{[j]}=\frac{1}{X_{i}}\left[\frac{\partial X_{i}}{\partial l} \mathrm{~d} l^{[j]}+\frac{\partial X_{i}}{\partial h} \mathrm{~d} h^{[j]}\right] \quad(j=1,2)
$$

where $\mathrm{d} l^{[j]}$ and $\mathrm{d} h^{[j]}$ represent how much the parameters $l$ and $h$ change when subjected to the stress $\mathrm{d} \sigma_{j}$ and can be expressed in terms of the applied stress $\mathrm{d} \sigma_{j}$ through the stretching stiffness constant as discussed below. Note that the superscript $[j]$ is used to denote that the stress is applied in the $O x_{j}$ direction. Note also that these systems do not shear for uniaxial loading in the $O x_{j}$ directions $(j=1,2)$, i.e. the shear coupling coefficients of this system are all equal to zero.

In analogy to Masters and Evans [6], one may define the stretching stiffness constants to be

$$
F=k_{\mathrm{s}}^{x} \delta x \quad x=h, l
$$

where $\delta x$ is the elongation of a rib with length $x$ after being subjected to a force $F$ along its length. In such cases, the on-axis strains in the $O x_{i}$ directions $(i=1,2)$ for loading by an infinitesimally small stress $\mathrm{d} \sigma_{1}$ in the $O x_{1}$ direction simplify to

$$
\begin{gathered}
\mathrm{d} \varepsilon_{1}^{[1]}=\frac{1}{k_{\mathrm{s}}^{l}}(\lambda+\sin (\theta)) \cos (\theta) \mathrm{d} \sigma^{[1]} \\
\mathrm{d} \varepsilon_{2}^{[1]}=\frac{1}{k_{\mathrm{s}}^{l}} \sin (\theta) \cos (\theta) \mathrm{d} \sigma^{[1]}
\end{gathered}
$$

whilst for loading in the $O x_{2}$ direction

$$
\begin{gathered}
\mathrm{d} \varepsilon_{1}^{[2]}=\frac{1}{k_{\mathrm{s}}^{l}} \sin (\theta) \cos (\theta) \mathrm{d} \sigma^{[2]} \\
\mathrm{d} \varepsilon_{2}^{[2]}=\frac{\cos (\theta)}{(\lambda+\sin (\theta))}\left(\frac{\sin ^{2}(\theta)}{k_{\mathrm{s}}^{l}}+\frac{2}{k_{\mathrm{s}}^{h}}\right) \mathrm{d} \sigma^{[2]} .
\end{gathered}
$$

Hence, the Young's moduli $E_{j}$ for loading in the $O x_{j}$ direction are given by

$$
\begin{gathered}
E_{1}=\frac{\mathrm{d} \sigma^{[1]}}{\mathrm{d} \varepsilon_{1}^{[1]}}=\frac{k_{\mathrm{s}}^{l}}{\cos (\theta)(\lambda+\sin (\theta))} \\
E_{2}=\frac{\mathrm{d} \sigma^{[2]}}{\mathrm{d} \varepsilon_{2}^{[2]}}=\frac{k_{\mathrm{s}}^{l}(\lambda+\sin (\theta))}{\cos (\theta)\left(2 K+\sin ^{2}(\theta)\right)}
\end{gathered}
$$

whilst Poisson's ratios $v_{j i}$ in the $O x_{i}-O x_{j}$ plane for loading in the $O x_{j}$ direction are given by

$$
\begin{gathered}
v_{12}=-\frac{\mathrm{d} \varepsilon_{2}^{[1]}}{\mathrm{d} \varepsilon_{1}^{[1]}}=-\left(\frac{\sin (\theta)}{\sin (\theta)+\lambda}\right) \\
v_{21}=-\frac{\mathrm{d} \varepsilon_{1}^{[2]}}{\mathrm{d} \varepsilon_{2}^{[2]}}=-\left(\frac{\sin (\theta)(\lambda+\sin (\theta))}{2 K+\sin ^{2}(\theta)}\right)
\end{gathered}
$$

where $\lambda=h / l$ and $K=k_{\mathrm{s}}^{l} / k_{\mathrm{s}}^{h}$ is the ratio between the stretching stiffness constants $k_{\mathrm{s}}^{l}$ and $k_{\mathrm{s}}^{h}$.

Since this system has zero shear coupling coefficients, from the compliance matrix of the system, the on-axis linear compressibility $\beta_{i}$ in the $O x_{i}$ direction may be obtained through the equations

$$
\begin{aligned}
& \beta_{1}=s_{11}+s_{12}=\frac{1}{E_{1}}-\frac{\nu_{21}}{E_{2}} \\
& \beta_{2}=s_{21}+s_{22}=\frac{1}{E_{2}}-\frac{\nu_{12}}{E_{1}}
\end{aligned}
$$

which simplify to

$$
\begin{gathered}
\beta_{1}=\frac{\cos (\theta)}{k_{\mathrm{s}}^{l}}(\lambda+2 \sin (\theta)) \\
\beta_{2}=\frac{\cos (\theta)}{k_{\mathrm{s}}^{l}}\left(\sin (\theta)+\frac{2 K+\sin ^{2}(\theta)}{\lambda+\sin (\theta)}\right) .
\end{gathered}
$$

The area compressibility $\beta_{12}$ may then be obtained using the equation $\beta_{12}=\beta_{1}+\beta_{2}$ [16],

$$
\beta_{12}=\frac{\cos (\theta)}{k_{\mathrm{s}}^{l}}\left(\lambda+3 \sin (\theta)+\frac{2 K+\sin ^{2}(\theta)}{\lambda+\sin (\theta)}\right) .
$$

It is worth adding here that using a method based on differentiation of the free energy by the strain, the same results were obtained.

\section{Results and discussion}

Typical plots for the Poisson's ratios and Young's moduli for a ratio $K / \lambda=0.001$ are shown in figure 2 together with the resultant plots of the linear compressibilities, shown in figure 3. These plots clearly show not only that negative Poisson's ratios (i.e. auxetic behaviour) can be exhibited by systems where $0^{\circ}<\theta<90^{\circ}$, which correspond to non re-entrant systems, but also that NLC along the $O x_{2}$ direction may be obtained from such truss systems in some particular configurations.

In particular, the equations and plots confirm that for loading in the $O x_{1}$ direction, the Poisson's ratios and moduli are in full agreement with what was discussed in earlier work on such systems [5, 6]. However, the in-plane Poisson's ratios and moduli for loading in the $O x_{2}$ direction may be better manipulated in the system presented here, which permits non-equivalent deformations in the vertical and inclined ribs, through careful choice of the ratio of the stretching stiffness constants $K$. 
(a)

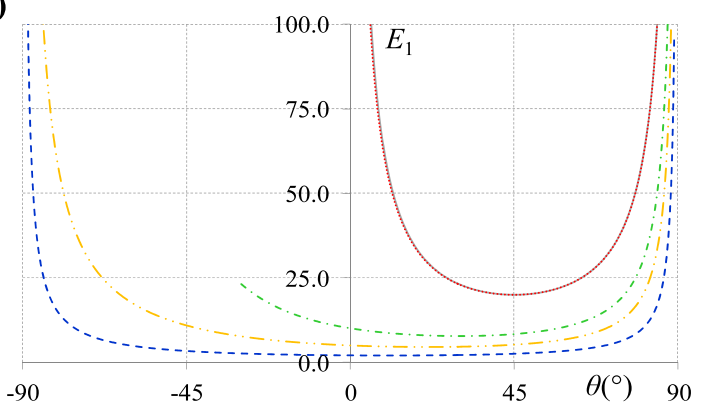

(b)

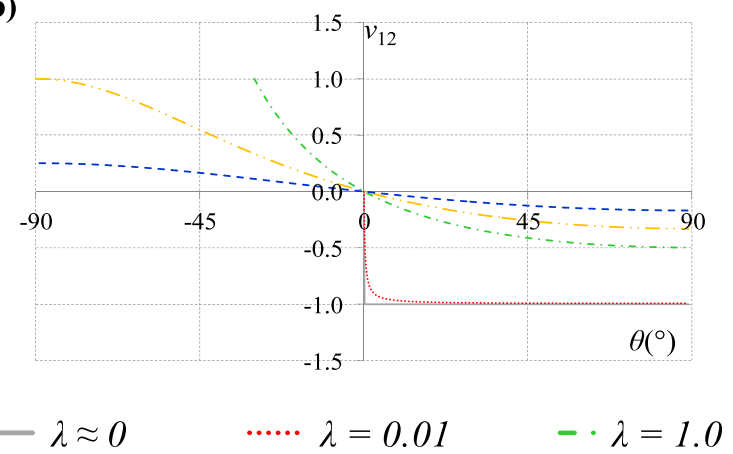

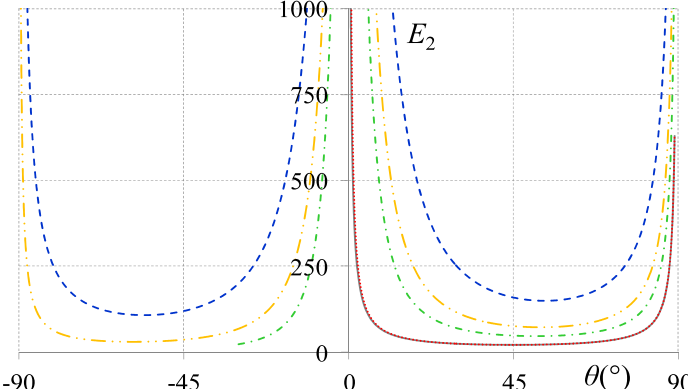

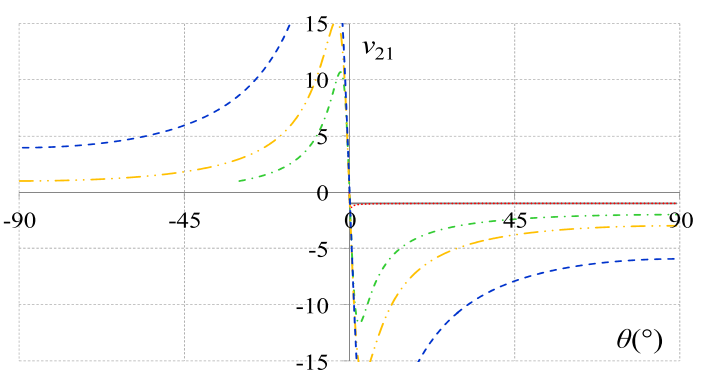

$-\cdots \lambda=2.0$

$-\lambda=5.0$

Figure 2. Plots of (a) Young's moduli and (b) Poisson's ratios against $\theta$ for systems where $k_{\mathrm{s}}^{l}$ is equal to 10 and $K / \lambda=0.001$. Note that systems with $\lambda \approx 0$ (i.e. $h=0$ ) correspond to wine-rack structures.

(a)

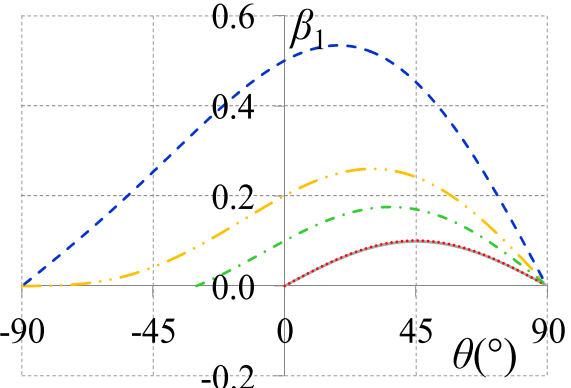

(b)

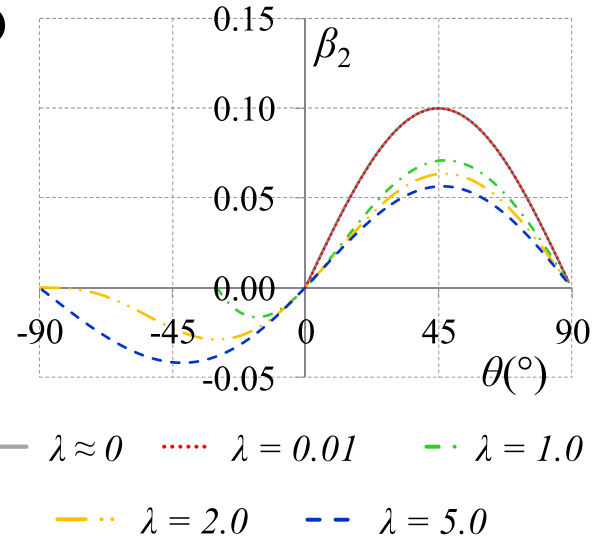

Figure 3. Plots of (a) $\beta_{1}$ and (b) $\beta_{2}$ against $\theta$ for systems where $k_{\mathrm{s}}^{l}$ is equal to 10 and $K / \lambda=0.001$. Note that systems with $\lambda \approx 0$ (i.e. $h=0$ ) correspond to wine-rack structures.

This difference, which arises due to the ratio between the stretching stiffness constants in the vertical and inclined ribs, may also give rise to anomalous compressibility properties. In fact, from the above equations and plots, it is evident that NLC can be obtained whenever there is a highly positive Poisson's ratio such that $v_{i j}>E_{i} / E_{j}$, a condition that can be achieved for re-entrant hexagonal truss systems made up of ribs of different stretching stiffness constants deforming solely through a constrained angle stretching mechanism. The presence and or absence of NLC may be explained by analysing the expressions derived above. In fact, with the constraint applied in order to have a physically realizable structure (equation (3)) and with analysis of equation (16) it is noticeable that under these conditions, the linear compressibility $\beta_{1}$ cannot be negative, irrespective of the value of the variables $h, l$ or $\theta$, since the term $(\lambda+2 \sin (\theta))$ is always positive for physically realizable structures. This, however, is not the case for the linear compressibility $\beta_{2}$, which can achieve negative values for a range of angles; this range of angles depends on the ratios $K$ and $\lambda$. Here, it should be noted that for negative linear compressibility $\beta_{2}$ to be observed, two conditions need to be satisfied simultaneously, namely that the range of angles has to be from $-90^{\circ}<\theta<0^{\circ}$, since $\sin (\theta)<0$ when $\theta<0^{\circ}$, and the condition that $\sin (\theta)<\left[\frac{1}{\lambda+\sin (\theta)}\left(2 K+\sin ^{2}(\theta)\right)\right]$. Thus, to increase the possible range of values of the angle $\theta$ where NLC is observed by satisfying the latter condition, one may either decrease the ratio of the stretching stiffness constants $K$ or else increase the $\lambda$ ratio (as in figure 3).

It should be emphasized that the above expressions still fulfil the demand that the overall area compressibility for physically realizable systems is positive since NLC in the $O x_{2}$ direction is always accompanied by a positive linear 
compressibility along the $O x_{1}$ direction such that the area compressibility of the system $\beta_{12}=\beta_{1}+\beta_{2}$ is always positive.

In fact, for physically realizable structures where $h+$ $2 l \sin (\theta)>0$ and hence $h / l=\lambda>-2 \sin (\theta)$ for the range of angles $\theta_{\min }<\theta<90^{\circ}$ where $\theta_{\min } \in\left[-90^{\circ}, 0^{\circ}\right], \cos (\theta)$ and $k_{\mathrm{s}}^{l}$ are always positive, hence if any negative area compressibility $\beta_{12}$ is observed, it would have to arise from the term $\lambda+$ $3 \sin (\theta)+\frac{2 K+\sin ^{2}(\theta)}{\lambda+\sin (\theta)}$ of equation (18). However it may easily be shown that

$$
\begin{aligned}
\lambda & +3 \sin (\theta)+\frac{2 K+\sin ^{2}(\theta)}{\lambda+\sin (\theta)} \\
& =\frac{\lambda^{2}+4 \lambda \sin (\theta)+2 K+4 \sin ^{2}(\theta)}{\lambda+\sin (\theta)} .
\end{aligned}
$$

Since for physically realizable structures $\lambda+2 \sin (\theta)>0$, then the denominator term $\lambda+\sin (\theta)$ is always positive since $\lambda>$ $\lambda / 2$ and $\lambda / 2+\sin (\theta)>0$, i.e. if any negative compressibility is observed, it would have to arise from the numerator term $\lambda^{2}+4 \lambda \sin (\theta)+2 K+4 \sin ^{2}(\theta)$, which is not possible. This can be shown through the worst case scenario, that is when $\theta$ has the lowest value, i.e. $\theta=\theta_{\min }$, in which case, using once again the condition for a physically realizable structure, one may simplify the numerator term by substituting $\lambda=-2 \sin \left(\theta_{\min }\right)$ such that

$$
\begin{aligned}
& 4 \sin ^{2}\left(\theta_{\min }\right)-8 \sin ^{2}\left(\theta_{\min }\right)+2 K+4 \sin ^{2}\left(\theta_{\min }\right) \\
& =2 K>0 .
\end{aligned}
$$

All this is very significant as it shows, for the first time, that if hexagonal truss systems are constrained to deform solely through a constrained angle stretching mechanism where the vertical ribs deform differently from the inclined ribs, by controlling the geometric parameters $h, l$ and $\theta$ along with their associated stretching stiffness constants, one can construct systems that exhibit negative linear compressibility along the $O x_{2}$ direction. This phenomenon could not be inferred from the earlier models such as those presented by Masters and Evans [6], as these earlier models did not permit such independence between $k_{\mathrm{s}}^{h}$ and $k_{\mathrm{s}}^{l}$ and instead they assumed that all ribs in the system had similar stretching properties, thus constraining $K$ to assume values of $K=$ $h / l=\lambda$. In such a special case, the generalized mechanical properties given in equations (10)-(13) become equivalent to the ones derived by Masters and Evans [6] and it can be shown that for this case NLC is not observed.

In fact, for the special case when $K=h / l=\lambda$ (figure 4), as was the case in Masters and Evans [6], it can be shown that $\beta_{2}>0$. In fact, equation (17) simplifies to $\beta_{2}=$ $\frac{\cos (\theta)}{k_{\mathrm{s}}^{l}}\left(\sin (\theta)+\frac{2 \lambda+\sin ^{2}(\theta)}{\lambda+\sin (\theta)}\right)$. For the range of angles $\theta_{\min }<\theta<$ $90^{\circ}$ where $\theta_{\min } \in\left[-90^{\circ} . .0^{\circ}\right], \beta_{2}$ is never negative since the term $\cos (\theta) / k_{\mathrm{s}}^{l}>0$ and so is the term which is multiplied with it since

$$
\sin (\theta)+\frac{2 \lambda+\sin ^{2}(\theta)}{\lambda+\sin (\theta)}=\frac{\lambda \sin (\theta)+2 \lambda+2 \sin ^{2}(\theta)}{\lambda+\sin (\theta)} .
$$

For physically realizable structures, the term $\lambda+\sin (\theta)$ is always positive, i.e. if any negative compressibility is observed, it would have to arise from the term $\lambda \sin (\theta)+2 \lambda+$ $2 \sin ^{2}(\theta)$, which is not possible since

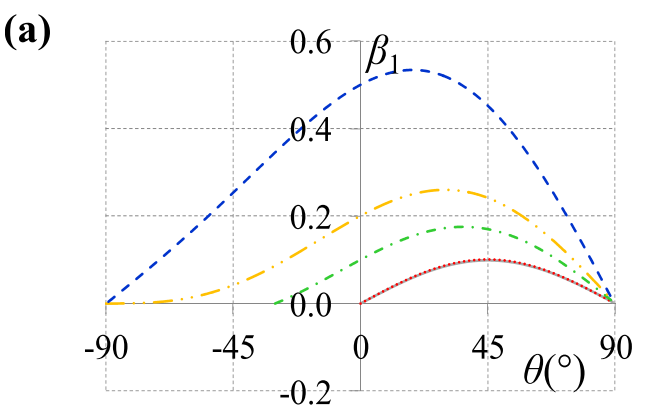

(b)

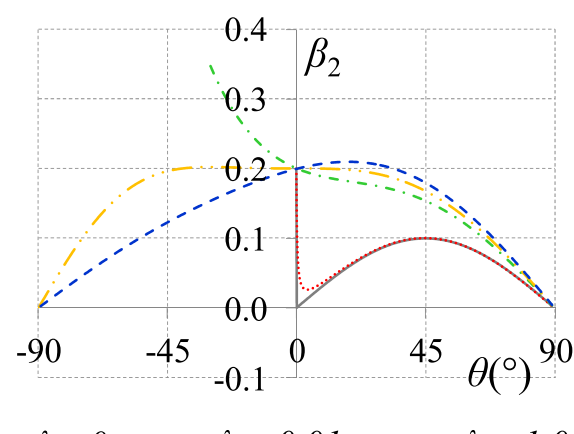

$$
\begin{gathered}
-\lambda \approx 0 \quad \cdots \cdots \lambda=0.01 \quad-\lambda=1.0 \\
-\cdots \lambda=2.0 \quad-\lambda=5.0
\end{gathered}
$$

Figure 4. Plots of (a) $\beta_{1}$ and (b) $\beta_{2}$ against $\theta$ for systems where $k_{\mathrm{s}}^{l}$ is equal to 10 and $K=\lambda$. Note that systems with $\lambda \approx 0$ (i.e. $h=0$ ) correspond to wine-rack structures.

$$
\begin{aligned}
\lambda \sin (\theta)+2 \lambda+2 \sin ^{2}(\theta)= & \lambda(\sin (\theta)+2) \\
& +2 \sin ^{2}(\theta)>0 .
\end{aligned}
$$

Using similar arguments, one can show that on increasing the ratio $K$, negative linear compressibility along the $O x_{2}$ direction is not observed, as illustrated in figure 5.

Furthermore, the equations and plots also show that for a wine-rack structure (i.e. $h=0$ ) NLC may not be observed when the structure deforms through the stretching mechanism discussed in this paper. This is due to the fact that for a wine-rack structure equation (17) simplifies to

$$
\beta_{2}=\frac{2 l \sin (\theta) \cos (\theta)}{k_{\mathrm{s}}^{l}}
$$

and with the constraint given in equation (3) for physically realizable structures, the resulting $\beta_{2}$ is always greater than zero.

Obviously, it must be noted that the above equations only apply if the structure deforms solely through a constrained angle stretching mechanism. In real systems, such stringent requirements may be difficult to achieve as other concurrent deformation mechanisms (e.g. flexing of the cell walls or hinging) may take place. In such cases, a more complex model which incorporates all these effects would be required for the system to be modelled in a realistic manner. Also, despite the fact that a novel mechanism for negative compressibility is being suggested here, this does not imply that the occurrence of NLC in non re-entrant hexagonal honeycombs described elsewhere will be enhanced through the constrained angle stretching mechanism proposed here. This is due to the fact 
(a)

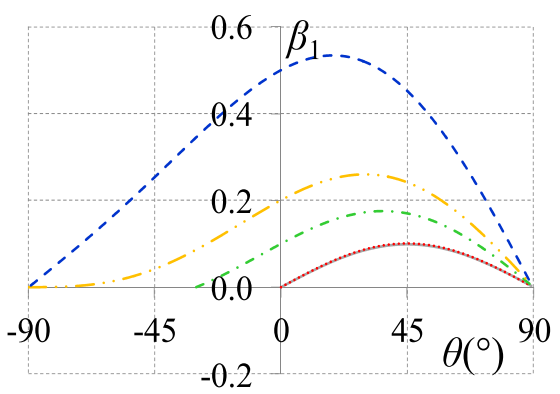

(b)

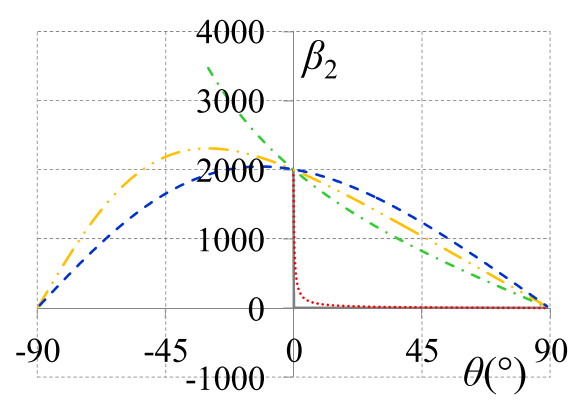

$-\lambda \approx 0 \quad \cdots \cdots \cdot \lambda=0.01 \quad-\lambda=1.0$

$-\cdots \lambda=2.0 \quad-\lambda=5.0$

Figure 5. Plots of (a) $\beta_{1}$ and (b) $\beta_{2}$ against $\theta$ for systems where $k_{\mathrm{s}}^{l}$ is equal to 10 and $K / \lambda=10000$. Note that systems with $\lambda \approx 0$ (i.e. $h=0$ ) correspond to wine-rack structures.

that the geometries that exhibit NLC with the mechanism proposed here are not the same geometries that exhibit NLC in the idealized hinging mechanism (i.e. the NLC in the case presented here arises from re-entrant geometries whereas for the hinging mechanism the NLC arises from non re-entrant geometries). Furthermore, it should be noted that in real systems, the stringent conditions required here may be difficult to achieve and a more complex model which incorporates other deformation modes would be realistic. Nevertheless, the model presented here would still be expected to give a good first approximation to the behaviour of such systems.

It is important to emphasize that the significance of the result reported here lies not only in the fact that NLC is a very rare property and it had never been reported so far from mechanisms of the form discussed here, but, more importantly, because materials that exhibit NLC are superior to conventional ones in various practical and niche applications [9-11, 19-21]. For example, it is envisaged that the mechanism proposed here may be of use in practical applications such as in high-pressure environments typically found in deep oceans. Materials and systems exhibiting negative compressibility can be superior to their conventional counterparts in such environments due to their potential to construct effectively incompressible structures. Such effectively incompressible structures can then be used in the manufacture of superior sensors and in optical telecommunication line systems [10]. Negative compressibility materials can also be used in extremely sensitive interferometric pressure sensors which detect pressure change by measuring changes in the optical path length (changes that are pressure-induced). On increasing the hydrostatic pressure on such devices, apart from an increase in density and hence an increase in their refractive index, volume compressibility occurs which typically results in a decrease in size in all directions. Such a decrease in size along the direction of light propagation results in a less sensitive way of measuring the change in the optical path length. However, if one uses materials that exhibit NLC along the direction of light propagation in such devices, one can improve their sensitivity since on increasing the pressure, densification still occurs (and hence a subsequent increase in the refractive index); however, the optical path length increases, hence the larger the extent of NLC of the material, the more sensitive the pressure sensor [10].

Further, before we conclude, it must be mentioned that although the versatile system discussed in this paper is a purely mechanical system it can also be engineered in a smart manner to, for example, replace the mechanical three-slot node shown in figure 1 to which the ribs are attached by a means of a micro-electro-mechanical system. This could permit control of the stretching stiffness constant by means of electromagnetic components, meaning that the macroscopic properties could be tuned by electromagnetism, possibly even remotely, a design which would make the presented system truly smart.

\section{Conclusion}

The analytical expressions derived here suggest that hexagonal truss systems made from ribs having different stretching stiffness constants can exhibit the rare and anomalous property of negative linear compressibility along a certain direction when they deform through a constrained angle stretching mechanism. The occurrence of such a property is exhibited when certain conditions are satisfied; these conditions have been elucidated above. It is hoped that the model presented here can inspire experimentalists to design materials and systems based on these concepts which may serve as a blueprint for the manufacture and design of man-made materials that exhibit such an anomalous property. Apart from this, the presented model may also serve to explain the occurrence of negative linear compressibility in naturally occurring materials that may be discovered in the future, as was the case with our earlier model on hinging systems, which is now known to be one of the more important mechanisms by which Nature can achieve this effect of NLC [18].

\section{Acknowledgments}

The support of the Malta Council of Science and Technology through their national R\&I programme as well as the support of the University of Malta is gratefully acknowledged.

\section{References}

[1] Lakes R S 1987 Foam structures with a negative Poisson's ratio Science 235 1038-40

[2] Evans K E, Nkansah M A, Hutchinson I and Rogers S 1991 Molecular network design Nature 353124 
[3] Gibson L J, Ashby M F, Schajer G S and Robertson C I 1982 The mechanics of two-dimensional cellular materials Proc. R. Soc. A 382 25-42

[4] Gibson L J and Ashby M F 1997 Cellular Solids: Structure and Properties 2nd edn (Cambridge: Cambridge University Press)

[5] Evans K E, Alderson A and Christian F R 1995 Auxetic two-dimensional polymer networks. An example of tailoring geometry for specific mechanical properties J. Chem. Soc. Faraday Trans. 91 2671-80

[6] Masters I G and Evans K E 1996 Models for the elastic deformation of honeycombs Compos. Struct. 35 403-22

[7] Scarpa F and Tomlin P J 2000 On the transverse shear modulus of negative Poisson's ratio honeycomb structures Fatigue Fract. Eng. Mater. 23 717-20

[8] Whitty J P M, Nazare F and Alderson A 2002 Modelling the effects of density variations on the in-plane Poisson's ratios and Young's moduli of periodic conventional and re-entrant honeycombs: part 1: rib thickness variations Cell. Polym. 21 69-98

[9] Baughman R H 2003 Auxetic materials: avoiding the shrink Nature $\mathbf{4 2 5} 667$

[10] Baughman R H, Stafstrom S, Cui C and Dantas S O 1998 Materials with negative compressibilities in one or more dimensions Science 279 1522-4

[11] Jaglinski T, Kochmann D, Stone D S and Lakes R S 2007 Composite materials with viscoelastic stiffness greater than diamond Science 315 620-2

[12] Lakes R S and Wojciechowski K W 2008 Negative compressibility, negative Poisson's ratio, and stability Phys. Status Solidi b 245 545-51
[13] Gatt R and Grima J N 2008 Negative compressibility Phys. Status Solidi Rapid Res. Lett. 2 236-8

[14] Grima J N, Attard D and Gatt R 2008 Truss-type systems exhibiting negative compressibility Phys. Status Solidi b 245 2405-14

[15] Fortes A D, Suard E and Knight K S 2011 Negative linear compressibility and massive anisotropic thermal expansion in methanol monohydrate Science 331 742-6

[16] Grima J N, Caruana-Gauci R, Attard D and Gatt R 2012 Three-dimensional cellular structures with negative Poisson's ratio and negative compressibility properties Proc. R. Soc. A 468 3121-38

[17] Barnes D L, Miller W, Evans K E and Marmier A S H 2012 Modelling negative linear compressibility in tetragonal beam structures Mech. Mater. 46 123-8

[18] Grima J N, Attard D, Caruana-Gauci R and Gatt R 2011 Negative linear compressibility of hexagonal honeycombs and related systems Scr. Mater. 65 565-8

[19] Cairns A B, Catafesta J, Levelut C, Rouquette J, van der Lee A, Peters L, Thompson A L, Dmitriev V, Haines J and Goodwin A L 2013 Giant negative linear compressibility in zinc dicyanoaurate Nature Mater. 12 212-6

[20] Gatt R, Caruana-Gauci R and Grima J N 2013 Negative linear compressibility: giant response Nature Mater. 12 182-3

[21] Grima J N, Attard D and Gatt R 2011 Unusual thermoelastic properties of methanol monohydrate Science $331687-8$

[22] Scarpa F and Tomlinson G 2000 Theoretical characteristics of the vibration of sandwich plates with in-plane negative Poisson's ratio values J. Sound Vib. 230 45-67

[23] Wojciechowski K W and Brańka A C 1994 Auxetics-materials and models with negative Poisson's ratios Mol. Phys. Rep. 6 71-85 